\title{
BMJ Open Qualitative, exploratory pilot study to investigate how people living with posterior cortical atrophy, their carers and clinicians experience tests used to assess vision
}

\author{
Michael Bowen, ${ }^{1}$ Harry Zutshi, ${ }^{1}$ Martin Cordiner, ${ }^{1}$ Sebastian Crutch, ${ }^{2}$ \\ Tim Shakespeare ${ }^{2}$
}

To cite: Bowen M, Zutshi H, Cordiner M, et al. Qualitative, exploratory pilot study to investigate how people living with posterior cortical atrophy, their carers and clinicians experience tests used to assess vision. BMJ Open 2019;9:e020905. doi:10.1136/ bmjopen-2017-020905

- Prepublication history for this paper is available online. To view these files, please visit the journal online (http://dx.doi. org/10.1136/bmjopen-2017020905).

Received 1 December 2017 Revised 12 December 2018 Accepted 17 December 2018

Check for updates

(c) Author(s) (or their employer(s)) 2019. Re-use permitted under CC BY-NC. No commercial re-use. See rights and permissions. Published by BMJ.

${ }^{1}$ Department of Research, The College of Optometrists, London, UK

${ }^{2}$ Dementia Research Centre, University College London, Institute of Neurology, London, UK

Correspondence to Mr Martin Cordiner; martin.cordiner@collegeoptometrists.org

\section{ABSTRACT}

Objectives To investigate the experiences and views of people living with posterior cortical atrophy (PCA), their family carers and healthcare professionals of vision assessment tests.

Design A qualitative investigation using video recordings of vision assessments, semistructured interviews and audio recordings of a focus group. Interviews and focus group used broad, open questions around the topic to prompt and guide discussion. Video and audio recordings were transcribed, manually coded and analysed using framework analysis.

Setting University College, London's Queen Square neurology centre provided the venues for all stages of the research.

Participants Participants living with PCA were one male and two females (age range 67-78 years). Health professional participants were a neurologist (male), two ophthalmologists (male) and an optometrist (female).

Primary and secondary outcomes (1) Experiences and attitudes of people living with PCA and health professionals to vision assessment tests, (2) views of health professionals and people living with PCA of whether some tests are more effective at discriminating between cortical vision problems and vision problems related to optical or ocular causes.

Results Patients were able to engage with and complete a number of tests. Their partners played a vital role in the process. Participants reported that simple, short tests were more effective than more subjective tests. Examples of tests that appeared to be more problematic for the patient participants were the Amsler Grid and visual field analysis.

Conclusions Although limited in scope and execution, the project suggests that some vision assessment tests are likely to support health professionals to discriminate between cortical and optical/ocular causes of visual impairment. It supports existing evidence that there are vision assessments that people with dementia can engage with and complete. We identify areas of importance for future research and make tentative suggestions for clinical practice.
Strengths and limitations of this study

- Small sample of patients took part in the study.

- Potential variation in the relative progression of posterior cortical atrophy between the participants may have been a confounding factor.

- Undertaken outside of usual clinical settings (due to multidiscipline approach), so patients might have performed differently in each discipline's normal clinical environment.

- Views on the experiences of both patients and practitioners in relation to each consultation captured separately, verbatim and on the day the consultations were undertaken.

- Multidisciplinary approach, incorporating optometric, ophthalmological and neurological screening tests.

\section{INTRODUCTION}

Posterior cortical atrophy (PCA) involves progressive visual dysfunction and a degeneration of the posterior brain's outer layer (the cortex). It is most commonly caused by Alzheimer's disease, although may also be caused by dementia with Lewy bodies, corticobasal degeneration or CreutzfeldtJakob disease. ${ }^{1}$ The visual dysfunction experienced can encompass aspects of visuospatial and visuoperceptual processing. Features of Balint's syndrome (eg, simultanagnosia, oculomotor apraxia) and of Gerstmann's syndrome (including acalculia and agraphia) are common. ${ }^{2-4}$

First described in 1988, consensus criteria for PCA have only recently been agreed ${ }^{5}$ and diagnosis is often delayed or absent. The fact that it often goes unrecognised means that a prevalence figure is hard to estimate (some studies have suggested about $5 \%$ of those diagnosed with early onset Alzheimer's 
disease may have $\mathrm{PCA}^{6}$ ). Most Alzheimer's disease cases appear in people over 65, but PCA tends to occur between 50 and $65 .^{78}$

People living with PCA often present to optometrists and ophthalmologists with non-specific visual problems, but unless the clinician specifically looks for the signs and symptoms of PCA, it may not be picked up. It is not uncommon for people living with PCA to report delays from first presentation with visual symptoms to final diagnosis of PCA of many years. Investigating how vision assessment is experienced by people living with PCA, and the health professionals' perspectives of conducting such assessments may offer insights into how to improve the process, and could provide scope for identifying tests that may be particularly useful in supporting clinicians to distinguish between visual symptoms with optical/ocular causes and those with cortical origins.

Individuals with PCA offer a unique perspective on the visual difficulties which may be experienced by many individuals with typical Alzheimer's, at a point when the memory, language and insight problems of the latter group limit their ability to communicate what they are experiencing. Also, the nature of cortical visual problems in PCA can confound the use of standard optometric assessments. For example, the majority of PCA patients have normal or near-normal visual acuity, yet may struggle with a standard Snellen letter chart because of a reduced effective field of vision, and so can find it easier to read smaller, rather than larger, fonts. They may also struggle with excessive visual crowding in their central vision, resulting in difficulty reading letters surrounded by other letters or clutter, ${ }^{9}$ another common trait of optometric testing charts.

\section{PURPOSE}

The complexities of both diagnosing $\mathrm{PCA}^{5}{ }^{10}$ and the reality that-given the complexities introduced by the cortical visual perceptual symptoms associated with PCAit may be complicated for optometrists and ophthalmologists to work with people living with PCA to find the most appropriate approach to correcting visual impairment (to produce the best possible visual experience) presented an opportunity for productive collaboration across the disciplines of optometry, ophthalmology, neurology and neuropsychology, to investigate the following research questions:

- How do people living with PCA experience various tests used to assess vision? What are the experiences of health professionals of administering these tests when examining people living with PCA?

- Are there particular tests for assessing vision that are more effective at discriminating between cortical vision problems and vision problems related to optical or ocular causes?

Qualitative methodologies, such as semistructured interviews, focus groups, ${ }^{11}$ and content analysis (or video/audio transcripts for example) offer an effective way to collect information about what patients think, how they think or why they may hold a particular view. Interactions between participants in groups can encourage participants to explore and clarify individual and shared perspectives and may support participation by people who may be reluctant to contribute their views in a more formal one-to-one scenario ${ }^{12}$

There is limited qualitative evidence from people living with PCA or the health professions involved in assessing vision for individuals in this group about the experiences of having vision assessed or assessing vision. In particular, there is little patient and clinician data about the experience of administering or being assessed using various standard tests. Focus groups have been used in a small number of studies to examine the general experiences of people living with conditions such as glaucoma, which require regular vision assessments. ${ }^{13} 14$

However, there is limited evidence relating to the opinions of patients living with PCA, or clinicians, about the tests used to assess vision. Anecdotal evidence suggests that patients dislike performing the visual field test, but no study has interviewed patients living with PCA in detail about their perceptions of the tests used by various health professionals to assess vision and visual perception. Such evidence could begin to shed light on how tests are experienced, and whether some tests may prove both more acceptable/accessible, and better able to support professionals in discriminating between cortical and optical/ ocular causes of visual problems.

This project was structured as an initial exploration of the qualitative experiences of people living with PCA and health professionals. The project was designed to gather rich qualitative data, although the limited sample size reduced the scope for definitive conclusions in relation to these questions to be reached. The intention was to explore the potential for gathering and analysing this type of data, with this participant group, with a view to informing the development of subsequent research. In addition to investigating the viability of such research, the project was intended to provide some insights into potential target tests and vision assessment methods of particular interest, to enhance and inform clinical practice and increase awareness of dementia-related cortical visual impairment through improved training and access to resources. It was also anticipated that it might enable further research to be more focused.

\section{METHODS \\ Participants}

Vision assessments and postassessment interviews took place over the course of a day at University College London's (UCL) Dementia Research Centre at Queen Square in London, in February 2016. This location was selected as it was familiar and accessible to participants living with PCA, convenient to the clinicians participating and had the scope to support the relevant equipment and filming required. A focus group was then held for 
the health professionals involved (as well as one other ophthalmologist invitee) in March 2016, to analyse footage selected from the vision assessments (footage was selected by HZ and MB initially, and reviewed by SC and TS before inclusion) and discuss a schedule of questions developed by the study team.

The study used purposeful sampling, whereby suitable participants living with PCA were selected by the UCL team from the Rare Dementia Support PCA support group membership ${ }^{15}$ (www.raredementiasupport.org), based on their ability and willingness to attend and on the need to have participants with a range of PCA presentations. Participants with PCA had a diagnosis consistent with the core clinicoradiological syndrome. ${ }^{10}$ Although all individuals presented with progressive decline in visual processing and relatively intact memory in the early stages, some impairments of episodic memory were apparent at the time of this study.

At present in the UK, many people living with early PCA are first referred into secondary care services by optometrists working in community settings. These referrals are frequently not identified as suspected PCA and due to the lack of any current referral pathways from primary care optometry to secondary care neurology services, the referral route is nearly always to the optometry or ophthalmology functions of hospital eye services. For these reasons the project aimed to include vision testing techniques from several different healthcare disciplines, to gain insights into possible differences at the various access and referral points. This meant that the logistics of the testing and interview schedule (in particular the time taken to complete each stage) restricted the number of participants to three people with PCA (one male, two females, age range 67-78).

Participants with PCA were given an information sheet with brief details of the purpose and programme of the day-to gather data about the experience of having vision and eye health assessed by a range of clinicians. A member of the research team provided the information verbally to participants with reading difficulties and checked with each participant that they had understood the information provided and answered any questions that the participant or their family member had. Written consent to participate in the research was received, along with written consent relating to the video recording of the examination sessions and interviews. Participants were informed in writing and reminded verbally on the day that they could withdraw from the project at any time. Each participant with PCA was accompanied by a family member throughout the processes of the day.

Three clinicians took part on the vision assessment day-an optometrist, a neurologist and an ophthalmologist (one female and two males), and they were given briefing information about the testing they would be asked to carry out and the post-testing interviews. Consent for participation and video recording of the assessments and audio recording of the focus group was received from each of the professional participants. The professional participants had varying experience of assessing vision in people with dementia or PCA. The optometrist had more than 20 years of experience in primary and secondary care settings, and had encountered numerous people living with dementia and some people living with PCA. The neurologist was an experienced consultant neurologist with more than 20 years of clinical and research experience working with numerous people living with PCA. The ophthalmologist was more recently qualified, and had encountered few people living with PCA.

The study conformed to the Declaration of Helsinki.

\section{Procedure}

Each patient participant completed three sequential vision assessments with an optometrist, ophthalmologist and neurologist, with their partner in attendance. After each assessment, the patient participant and their partner completed a brief interview, as did each clinician.

In advance of the testing day, semistructured interview schedules were developed for the postexamination interviews with the clinicians and people with PCA. Following the day of vision assessments, $\mathrm{HZ}$ and $\mathrm{MB}$ prepared a schedule of questions/topics for the clinicians' focus group (which took place two weeks after the vision assessment day to allow time for video footage to be reviewed and selections made for presentation to the focus group), informed by the themes arising during the eye examinations and postexamination interviews.

\section{Vision assessment protocol}

Each of the three health professionals was asked to assess the vision of each of the three participants in a manner that followed as closely as possible the methods they would use in their usual clinical practice.

These broad test protocols equated to: optometristprimary care general ophthalmic services sight test ${ }^{16}$; ophthalmologist-general secondary care hospital eye service general referral (refraction clinic) vision assessment; neurologist-the visual perceptual elements of a routine neurological examination.

Each clinician was provided with as much of the equipment for assessing vision that they would usually have available.

Equipment available was as follows:

- Hand held slit lamp (Keeler).

- Tonometer - CT-80 (table mounted).

- Field screener - Henson 9000.

- Ophthalmoscope.

- Retinoscope.

- Indirect ophthalmoscope (Keeler).

- Prism bar.

- Cross-cyl test lenses.

- Trial frame.

- Trial frame lens set.

- Focimeter (Pentax).

- Volk lens.

- 20D lens 
Tests/charts:

- Standard Snellen chart.

- Thompson Software Electronic test chart software (running on an Apple iPad) -including cross-cyl and near reading.

- Near reading test chart - cards.

- Ishihara colour vision test cards.

- Frisby stereo test chart.

- Cardiff cards or Teller visual acuity cards.

- The Queen Square Screening Test for Visual Deficits (The Blue Book). ${ }^{16}$

- The Queen Square Screening Test for Cognitive Deficits (The Green Book). ${ }^{16}$

As the primary objective of the study was not to investigate a specific vision assessment procedure, or test sequence, the health professionals were not given a specific sequence for test elements. They were asked to take the approach to vision assessment/sight testing that they would usually follow in their practice setting.

These were the agreed key assessments that the health professionals suggested would generally be included in their usual assessments:

Neurologist's assessment:

- Medical history.

- General examination.

- Eye signs (visual fields on confrontation, eye movements).

- Limb signs.

Ophthalmologist's/optometrist's assessment:

- Medical history.

- Ophthalmoscopy.

- Retinoscopy.

- Slit lamp examination.

- Subjective and objective refraction.

- Convergence.

- Ocular motility.

- Pupil reflexes.

- Intraocular pressure (tonometry).

- Visual fields.

- Accommodation.

The order of optometry, ophthalmology and neurology assessments was varied between participants in an ABC BCA CAB design, with each assessment followed by an interview.

\section{Interview and focus group procedures}

The interview and focus group schedules were developed following initial discussions within the study team. The topics identified and included within the schedules served only as a guide for the interviews and focus group. The order in which topics were addressed in interviews was not rigidly applied and question wording was not prescribed in advance. Where considered helpful, prompts were used by the interviewer/focus group facilitator to introduce topics and to encourage participants to expand on their comments. However, the core of the discussion came from the participants and care was taken to use open questions and to avoid unduly leading the conversations.

Although the patient participants were aware that they were going to have their eyes examined and vision assessed by the clinicians, they were not made explicitly aware of the focus of the study being to identify how tests were experienced and whether any tests were particularly good or bad when being used with someone with PCA. All interviews, and the facilitation of the clinicians' focus group, were conducted by one of the investigators (HZ). The professionals' focus group was cofacilitated by MB. The interviewer and participants had not met each other prior to the testing day, so introductions were made prior to the first interviews. In addition to the video recordings, the interviewer took field notes during the interviews. This note taking was intentionally kept to a minimum to enable the interviewer to attend as fully as possible to the interviews. During the focus group other members of the investigation team took notes to free the facilitator to focus on the discussion.

Each postexamination interview lasted around $5 \mathrm{~min}-$ these interviews were intentionally kept brief to manage the time/energy demands of a long day of testing for the participants. Each filmed examination session lasted approximately 20-30 min. The clinicians' focus group lasted for about three and a half hours, with a $15 \mathrm{~min}$ break in the middle.

Consolidated Criteria for Reporting Qualitative Research guidelines were followed in the design and reporting of the study. ${ }^{17}$ A Systematic Reporting of Qualitative Research checklist was completed to ensure that the final paper complied with these guidelines. ${ }^{18}$

\section{Patient and public involvement}

The research question was developed following patient and public data collected during the PrOVIDe study (708 participants), ${ }^{19}$ and also from discussions with members of the UCL PCA Support Group at one of their regular meetings (60 attendees).

Potential participants (members of the UCL PCA Support Group) were asked about the feasibility of the design, and any specific concerns and interests prior to the final design being confirmed.

Patients were not directly involved in the collection or analysis of data in this project, nor in the process of recruiting participants.

All those who participated were informed of the outcomes of the vision assessments where these indicated the need for further investigation/referral. Participants will receive a copy of the final report and any publications, and these will also be shared with the wider membership of the PCA Support Group and other relevant patient networks. Notice of papers will be given in the Alzheimer's Society patient publication.

This study was not a randomised controlled trial, so there was no trial-related requirement to assess the burden of the intervention on the patients. However, the team gave careful thought to the schedule for the day, 
Table 1 Framework technique used for data analysis

1. Familiarisation

Manuscripts are read and re-read independently by investigators.

$\begin{array}{ll}\text { 2. Identifying thematic } & \text { Themes are identified and } \\ \text { framework } & \text { then reviewed jointly and } \\ & \text { a refined/condensed set of } \\ \text { themes agreed on. }\end{array}$

3. Coding/indexing

Codes are applied to the data systematically by both investigators independently. Coding is then reviewed and discussed until final consensus on coding is reached.

4. Charting
\[ \begin{array}{l}\text { Data is rearranged in line } \\ \text { with thematic content in } \\ \text { a manner that supports } \\ \text { cross-case and within-case } \\ \text { analysis. }\end{array} \]
5. Mapping and interpretation
$\begin{aligned} & \text { Data is interpreted } \\ & \text { and conclusions and } \\ & \text { recommendations drawn. }\end{aligned}$

the provision of breaks and rest periods and made clear to participants that if the process was too tiring that they could rest or drop out at any time.

\section{ANALYSIS}

All vision tests/eye examinations and postexamination interviews were video recorded. The clinician focus group was audio recorded. The dialogue from the video and audio recordings was transcribed and reviewed by the investigators. In a small number of instances certain words were inaudible on the recordings, so field notes were used to account for any unclear information in those sections. All transcripts were pseudonymised.

The project followed an approach that was broadly constructivist and founded in the concepts of grounded theory. Data were analysed by two of the authors (MB and HZ) independently using framework analysis ${ }^{2021}$ as shown in table 1. An inductive approach was taken to coding and analysis. Each investigator read and re-read the transcripts and manually identified the key themes from the data. Once the investigators had both completed their independent theme identification, they met to review respective themes and organise the thematic framework, condensing and refining the categories that had been identified and identifying additional themes for exploration. Any differences of opinion regarding the relative importance of themes, or the meanings of sentences were discussed until a consensus was reached.

\section{Findings}

Initial coding was completed according to the themes identified and agreed following stages 1 and 2 of the framework in table 1. During coding additional themes were identified. There were also occasions where it became clear during the coding process that themes initially considered distinct were actually either a single theme or a theme and very closely linked subtheme. Themes and subthemes are summarised in figure 1 .

\section{RESULTS}

All of the participants were able to complete the full sequence of vision assessments with each of the participating professionals. However, within each assessment there was variation regarding the participants' ability to complete individual tests. Although $45 \mathrm{~min}$ was allowed in the schedule, with the exception of the neurologist, the professional participants found it difficult to complete the tests within the time, or found that the patient participants were finding the testing tiring.

\section{The test experience}

Clinicians reported that it was difficult to take a reliable history because of patient memory problems. They found that the simple, short tests appeared to work the best. Tests that included too many variables appeared to be less readily administered and were agreed to be likely to be less effective with these three patients. Examples of tests that appeared to be more problematic for the patient participants were the Amsler Grid $^{22}$ and visual field analysis. Other optometric, ophthalmic and neurological tests were generally reported by the clinicians as appearing to be more effective; however, more subjective tests such as colour vision, depth perception and visual acuity were reported by patient participants as being, or seemed to clinician participants to be, more difficult for the patient participants. This was reported as apparently being due to either difficulty in understanding and/or retaining the instructions or visuoperceptual problems in completing the test, or some combination of these. A neuropsychological test using full and fragmented letters or images (see figure 2) appeared to offer potential as a screening test to discriminate between optical/ocularvision problems and cortical visual deficits. This type of test had the benefit of being short and simple. The professional participants agreed that this would be a good target for further research.

Clinicians noted that patients were affected by their involvement in one test after another. Fatigue was definitely a factor by the end of the day and within the test process. Patients would become more distracted, for example when their second eye was being tested. This meant that the time that testing took was significant. Too long and the patient may become too tired to continue without a break. Also, the testing process was particularly challenging for patients as it explored skills that they were once proficient in such as reading, but now find a struggle. Testing provided constant reminders of this.

All three patients attempted reading. This is challenging for the patients as it is an aspect of real life 


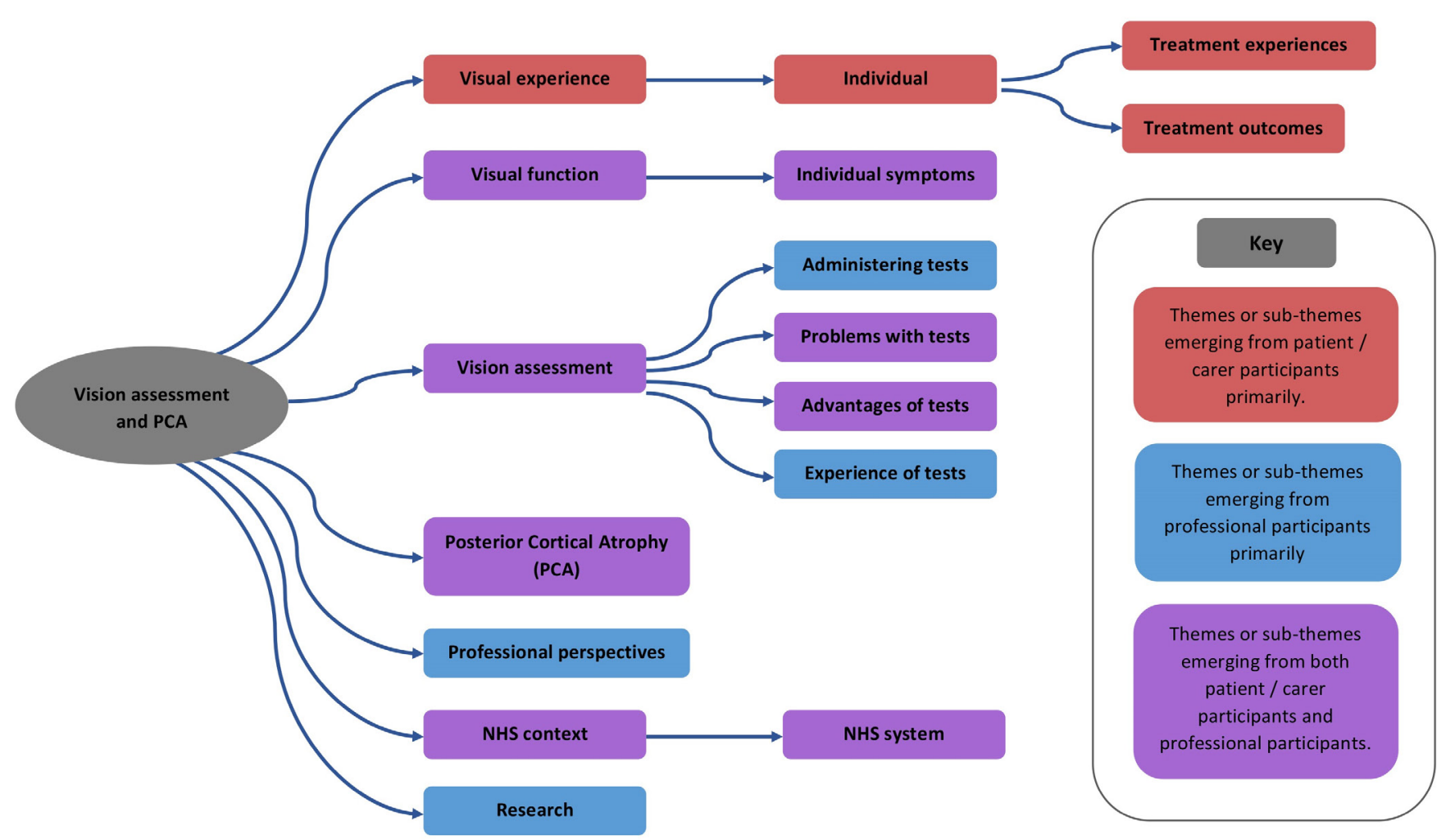

Figure 1 Themes and subthemes identified. NHS, National Health Service.

they are concerned about anyway. I also checked to see if using your finger as a guide helped with reading but it did not. (Clinician interview 12.2)

Patients gave two reasons why they had volunteered to be involved in the testing day. First, it was a chance to

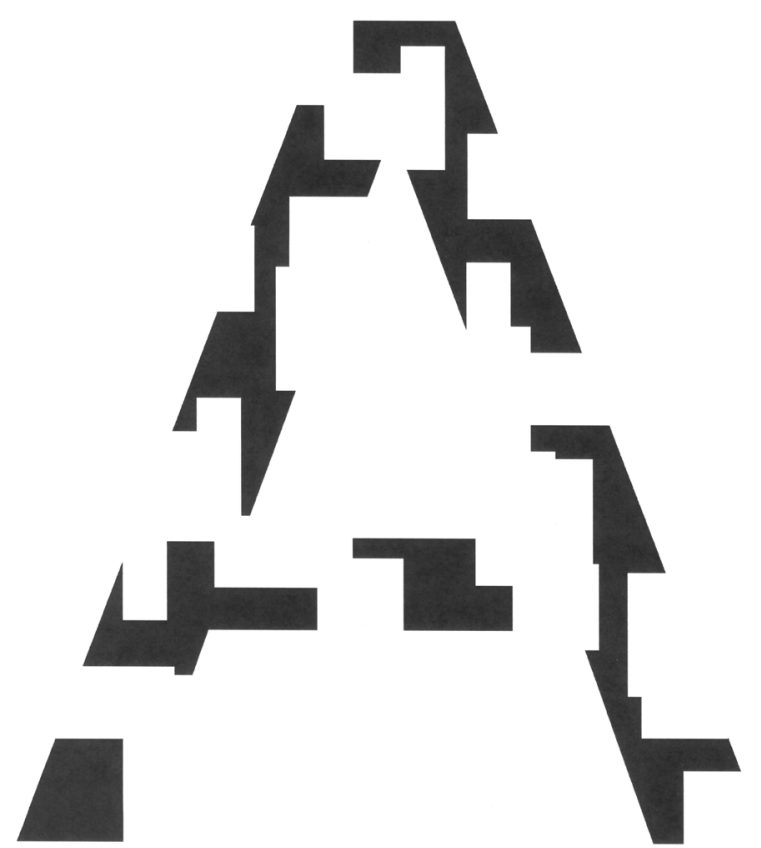

Figure 2 An example of a fragmented letter, in this case the letter ' $A$ '. contribute to research into a greater understanding of PCA, as patients had had difficulties in gaining an accurate diagnosis of their condition in the first place. Second, it was a chance to find out more up-to-date information about how their disease was progressing and to check their vision.

Patients recognised that vision assessments were generally necessary.

It is as if I have been smacked in the face ... that sort of feeling that you get when you have blown your nose too hard or been hit in the nose. So that is a physical feeling. It's almost like little hooks being pulled around the eye, it's quite hard work. (Patient interview 3.4)

Although they all remained positive about the process of repeated vision assessments on the day, and were made aware of the fact that they could stop at any point during the day they also found it uncomfortable and emotional at times, as it focused on what they were not able to do. As well as reporting tiredness, it could be experienced as physically unpleasant as well.

Patient responses varied greatly across the tests. Some they found easy to do, some were difficult, while one or two they could not do at all. All patients reported positively on the clarity of explanation of the test elements by each clinician. They welcomed the fact that key information was repeated at intervals. These reminders of the key project information included research team members 
checking that participants were happy to continue and ensuring that they were aware that they could take additional breaks between the sessions if they wished to. Tea/ coffee and juice/water were available to participants on request, and were regularly offered.

Patients' partners played a vital role in the testing process. This role went beyond encouragement and support. It was about helping and prompting patients where they had memory lapses. They had shared patient frustrations when it had been difficult for clinicians despite many tests to diagnose PCA in the first place. Patients could turn to their partners for assurance during the tests, which could be given simply as a nod of encouragement or the prompt of a correct word.

The real crux of it is to recognise the move from eyes to brain. I am not sure still from the sort of ophthalmic tests we had, which are fairly standard eye tests that even a competent ophthalmologist would pick up necessarily that it is a brain disorder, that it is to do with processing the information. (Partner comment in patient interview 6.8)

\section{Identifying cortical perceptual problems}

The clinicians reviewed their experience of working with patients with PCA. They argued that it is important to look at two different aspects of care, prediagnosis and postdiagnosis. Prediagnosis there were real concerns about a wide range of clinicians who could potentially be involved, but who may find it difficult to identify suspect-PCA or to discriminate between optical/ocularvision problems and cortical perceptual issues.

They come in saying I've got a problem with my eyes, I can't see things and we do our examination and say, actually no, we can't really find any deficit at all, back you go to your GP and really the diagnosis would potentially be missed. (21 merged coding)

They could include optometrists, ophthalmologists, neurologists and general practitioners. If it was possible to develop a simple test or series of tests to give an indication that a visual perceptual deficit or condition such as PCA may be involved, then this would be a significant step forwards and avoid a situation where patients visit a number of clinicians without anyone coming up with a firm diagnosis. This would also have potential for primary care settings as well. One clinician also thought it would be useful to involve orthoptists.

Post diagnosis it is still very important that the patient is able to access primary eye care so that they get monitoring of their general eye health and accurate correction of vision defects. (76 merged coding)

Patients thought that they fell between different clinical disciplines, going from one to another with no definitive diagnosis. Patients often reported a number of common symptoms. These included not being able to read dot matrix signs in the underground or on buses, and dislike of shiny surfaces and down escalators. A particular frustration was the ability to read, which might come and go in some patients, or be fully lost for others.

I was constantly being bumped from pillar to post either at the hospital ophthalmic department or another trying to work out why I couldn't read properly and everything was falling off...things would slide off the page, I would say like icing off a cake. (Patient interview 3.9)

As a result of this, a key priority for patients and their partners was that appropriate systems were in place to enable early identification of PCA by primary and secondary care professionals. This meant that a consistent approach was needed across optometry, ophthalmology and neurology, with clear, effective and prompt communication and referrals between clinicians.

\section{Learning from the tests}

Clinicians reviewed the learning from the project testing through detailed discussions in the focus group. There were a number of issues which emerged from this. Using a chart with lines of letters was far less effective than presenting patients with images of single letters. Multiple lines often caused patients to mix up letters on different lines. A simple test which contrasted full and fragmented images or letters was agreed to be the test that provided clearest evidence of PCA, or symptoms of other cortical vision problems, as patients could identify the full image but not the fragmented one. This worked with a letter or another object as the image. Another test was found to be to use photographs of common objects, but from unusual angles. Patients also experienced other symptoms which while not necessarily unusual for people living with PCA or other cortical visual problems, would be relatively uncommon in most primary care eye health settings.

For example, one said she could identify a small crumb on the floor but yet not see a glass on the table. One neurological test looked at visual disorientation. The patient was asked to grasp the clinician's finger, but was often unable to do so. It is not uncommon for visual field defects to be confused with problems and disorders of spatial cognition (such as simultanagnosia) ${ }^{23}$ which may lead to eye health professionals missing a cortical problem such as PCA. For example, people with simultanagnosia may have serious problems performing perimetric tests (and thus appear to have limited visual fields), while their visual field may be intact in terms of their optical system and ocular health. ${ }^{24}$

I ask patients to grab my finger. This can look like a field defect, but it is not. Patients can see the hand and can copy the hand movement, yet cannot locate the finger in space. There is an unusual visual field and visual disorientation. (Clinician interview 12.4)

One clinician noted that it would be useful to include a routine slit lamp investigation with the tests in order to help determine the presence/absence of retinal 
pathology. Patient participants did however report that the slit lamp examination was one of the most unpleasant parts of the optometric assessment. Another thought that it might be worth trying other field test approaches such as confrontation fields or some of the more recent tabletbased field tests. Patients experienced particular problems with the visual field analysis. One patient could not see the light at all, while it 'came and went' for another patient. It is possible that this was due to optic ataxia, which is not uncommon among people living with PCA, but in primary eye health practice or general ophthalmology clinics this might not be readily seen as the most obvious explanation for such an observation. ${ }^{25}$

Can you see the light?' and I had to say no, I couldn't.

He said, "Can you tell me where the light is?" and I said, "What light?" (Patient interview 4.5)

Patients and partners noted that there could be a cumulative effect of testing which involved things that they could not do, or skills they had lost. This increased fatigue and made concentration harder. Sometimes a break was needed.

Patients demonstrate awareness that their own memories could be erratic and unreliable. They were also aware that they could have good and bad days. These factors seemed to be driving the patient and carer view that the test process needed to be flexible enough to accommodate these different patient responses and needed to take account of their fatigue and frustration with not being able to do things that were once normal everyday skills.

Two of the clinicians indicated that they had greater confidence in running tests for people with PCA after the testing with the three patients. They both had experience of previous patients where it was harder to carry out testing. The clinicians were interested to discuss how what they had learnt from the research could be put into practice within their own work settings. Making changes such as splitting testing into two parts could help to alleviate some of the fatigue and distraction. This would not just apply to PCA patients, but also those with other forms of dementia.

The optometrist raised the issue of the length of the average sight test during a standard day's practice. The traditional $20 \mathrm{~min}$ test was clearly insufficient for what was involved. She found a good pattern was to alternate $30 \mathrm{~min}$ and $45 \mathrm{~min}$ tests, allowing scope for patients with more complex needs (56/57 merged coding).

Discussions between clinicians reflected patient concerns about the time that it took to get a correct diagnosis of PCA. Some issues reflected wider problems about the lack of training to work with patients with dementia. A benefit of the research process was that it had brought together optometry, ophthalmology and neurology. However, there was a lack of awareness throughout the different disciplines about PCA and this would need to be tackled in the future. The involvement of primary care, particularly GPs would also be vital to this.
People aren't making the diagnosis always and they're getting misdiagnoses or the patient's being pigeon-holed in the wrong place. (76 merged coding)

\section{Future research implications}

Clinicians expressed particular interest in the implications of this project and its exploration of tests and the testing process. This was discussed during their one to one interviews, but particularly in the focus group. It was apparent that there were two broad areas for taking the research forwards. First, it was important to gain greater clarity about the numbers of patients with PCA within the broader spectrum of people with dementia. Second, there needed to be greater awareness of PCA by making use of development opportunities across the different professions, and data about the current level of understanding would provide a baseline against which to measure educational interventions.

Greater clarity about numbers could be achieved by re-analysing previous tests which have been used on a larger scale and included full and fragmented letters as part of the wider test. It may also be possible to add this element to new research as well. However, discussion emphasised that looking at numbers alone was not enough. If clinicians could not recognise PCA, they would not be able to diagnose it from the sometimes contradictory information they may come across from testing patients.

The low hanging fruit as far as a simple research question goes is: what are the three or four things which if you've got two or more of them then you are really thinking, it's not just an eyesight thing it's a brain thing? A test like that could be done in 30 s. (62 merged coding)

It was suggested that one way to establish a baseline of understanding of dementia and PCA in particular would be, 'to run some short surveys with medical students across optometry, ophthalmology and neurology to gain a clearer understanding of current levels of awareness' (95 merged coding). This would help to develop such baseline data across the relevant professions.

Previous research ${ }^{19}$ has shown that there is a strong association between visual impairment and the likelihood of being in residential care. The prevalence of visual impairment from all causes was found to be more than 2.5 times greater in residential home settings, even allowing for age and severity of dementia. Improving people's visual functioning will help their quality of life and increase their chances of staying out of residential care.

I'm always worried that we work in a specialist centre and we get people with particular diagnoses and we've got very little idea of how representative our sample is of the rest of the world. (103 merged coding)

Further research into screening tests for PCA is vital, and was considered to be an important follow-up to this 
pilot research by all the clinicians. This would involve identifying a small group of tests, such as the full and fragmented letters test, and trying them on different groups of patients. However, a significant factor with any screening test could be the number of false positives, which it was suggested might lead to too many referrals to neurology. More elaborate research follow-up could include running tests with a group of patients with PCA, a group with typical, memory-led Alzheimer's disease and an appropriate control group (or groups). There may also be other outcomes from existing surveys and other research that has already been completed, which could be aggregated into a literature review.

Existing research has shown that there is a stark difference in the mean onset age for PCA compared with Alzheimer's disease. A participant pointed out that for PCA this age is 59, while it is at least 20 years later for Alzheimer's disease (94 merged coding).

\section{Limitations}

Clinicians expressed some reservations about the fact that tests had only been tried on three patients. It may be important to look at a wider range of patients as this could highlight other issues that might not be apparent from this small sample. Given the relatively early average age of onset of PCA, the ages of the participants in this explorative study means that additional data from people living with earlier PCA would be important to gather in subsequent research.

The neurologist thought that the project's test process could have benefited from the use of a wider range of screening tests. It is possible that some of these would prove more effective than others. Also, one clinician thought that it had not yet been possible to test the limits of what the patients could manage within the requirements of the research setting (105 merged coding) and the resources for testing available, which had not been as extensive as in their usual clinical settings.

However, there was agreement among the clinicians that patients had done much better on the tests than might be expected, given their complex range of problems (59 merged coding). This is positive as it provides some further support for the finding that many people living with dementia could complete most of the key elements of a standard sight test. ${ }^{19}$

\section{CONCLUSIONS}

A simple test which contrasted full and fragmented images or letters was agreed to be the test that provided clearest evidence of PCA, or symptoms of other cortical vision problems, as patients could identify the full image but not the fragmented one. More generally, the clinicians felt that simpler, shorter objective tests appeared to be generally more accessible to the patient participants than more complex, longer or more subjective ones. The benefit of support from partners within the examination environment itself was also clear.
A key priority for patients and their partners was that systems were in place to facilitate early identification of cortical perceptual problems and to have these referred into the appropriate secondary care service to enable a clear diagnosis of PCA (or other neurological condition causing the problem) to be confirmed. This meant that a consistent approach was needed across optometry, ophthalmology and neurology, with effective and prompt communication and referrals between clinicians, to prevent excessive and unnecessary delay in diagnosis. These concerns were echoed by the clinical professionals who acknowledged the difficulty many health professionals would currently be likely to have in making clear discriminations between optical/ocularand cortical vision problems.

The test process needs to be flexible enough to accommodate atypical patient responses, and needs to take account of these patients' fatigue in general, and also their frustration with not being able to do things that were once normal everyday skills.

The professional participants in this explorative research project strongly agreed that future research should clarify numbers with PCA, establish cross-profession knowledge and skills in this area, and work on further screening tests for PCA, and although limited in scope and execution, the project supports existing evidence that there are suitable eye examination tests that people with dementia can engage with and complete.

\section{RECOMMENDATIONS}

The outcomes from this project suggested that there were a number of recommendations which could be taken forward.

1. Refine and simplify optometric and ophthalmological tests to make them more effective for patients with PCA or dementia more widely, and undertake research to find out how these work in practice with larger and more varied cohorts of patients.

2. Include the full and fragmented letters test and related tests from the Queen Square Screening Test for Visual Deficits ${ }^{16}$ used by neurologists as part of the research outlined in point 1 , and examine their effectiveness in differentiating between optical/ocularand cortical vision problems (caused by conditions such as PCA) in order to develop understanding of their potential to aid clinicians in primary and secondary care settings to discriminate between visual problems with optical/ ocular causes and those with cortical causes.

3. Develop professional learning materials to raise awareness of PCA.

4. Develop concise resources for patients with dementia so they can make the most of their eye test.

5 . Review previous research to identify what indications there are about the prevalence of PCA in the UK.

Acknowledgements The research team would like to thank all of the participants in the project: people living with PCA, family members who supported them to 
take part and the clinicians who took part. We would also like to acknowledge the contributions made by members of the UCL PCA Support Group, who provided advice on the initial development of the research question and on the feasibility of the proposed approach to the project/project design. The team would also like to acknowledge the support provided by Topcon UK through the generous Ioan of items of optometric equipment. The generous assistance of Mycal Miller, who filmed all of the participant interviews and sight testing is also gratefully acknowledged.

Contributors All of the co-authors were involved from the outset in the design and development of the project and the research protocol. MB codrafted the manuscript with $\mathrm{HZ}$ and reviewed and approved the final draft for submission. MC redrafted the manuscript and approved the final draft for submission. SC reviewed drafts of the manuscript and approved the final draft for submission. TS reviewed drafts of the manuscript and approved the final draft for submission. $\mathrm{HZ}$ undertook the interviews and led the Focus Group as part of the project, codrafted the manuscript and reviewed and approved the final draft for submission.

Funding This work was funded by the College of Optometrists and University College London. TS was supported by an Alzheimer's Research UK Research Fellowship. SC was supported by a grant from ESRC/NIHR (ES/L001810/1).

Competing interests None declared.

Patient consent for publication Not required.

Ethics approval The study was approved by the Queen Square Research Ethics Committee.

Provenance and peer review Not commissioned; externally peer reviewed.

Data sharing statement The unedited transcripts of the interviews are held by the College. They are not currently publicly available.

Open access This is an open access article distributed in accordance with the Creative Commons Attribution Non Commercial (CC BY-NC 4.0) license, which permits others to distribute, remix, adapt, build upon this work non-commercially, and license their derivative works on different terms, provided the original work is properly cited, appropriate credit is given, any changes made indicated, and the use is non-commercial. See: http://creativecommons.org/licenses/by-nc/4.0/.

\section{REFERENCES}

1. Mendez MF, Ghajarania M, Perryman KM. Posterior cortical atrophy: clinical characteristics and differences compared to Alzheimer's disease. Dement Geriatr Cogn Disord 2002;14:33-40.

2. Rizzo M, Vecera SP. Psychoanatomical substrates of Bálint's syndrome. J Neurol Neurosurg Psychiatry 2002;2002:162-78.

3. Tang-Wai DF, Graff-Radford NR, Boeve BF, et al. Clinical, genetic, and neuropathologic characteristics of posterior cortical atrophy. Neurology 2004;63:1168-74.

4. Crutch SJ, Schott JM, Rabinovici GD, et al. Alzheimer's Association ISTAART Atypical Alzheimer's Disease and Associated Syndromes Professional Interest Area. Consensus classification of posterior cortical atrophy. Alzheimer's \& Dementia 2017:30040-7.

5. Snowden JS, Stopford CL, Julien CL, et al. Cognitive phenotypes in alzheimer's disease and genetic risk. Cortex 2007;43:835-45.
6. Galton CJ, Patterson K, Xuereb JH, et al. Atypical and typical presentations of Alzheimer's disease: a clinical, neuropsychological, neuroimaging and pathological study of 13 cases. Brain 2000;123:484-98.

7. Schott JM, Crutch SJ, Carrasquillo MM, et al. Genetic risk factors for the posterior cortical atrophy variant of Alzheimer's disease. Alzheimers Dement 2016;12:862-71.

8. Yong KX, Shakespeare TJ, Cash D, et al. (Con)text-specific effects of visual dysfunction on reading in posterior cortical atrophy. Cortex 2014;57:92-106.

9. Zakzanis KK, Kielar A, Young DA, et al. Neuropsychological differentiation of late onset schizophrenia and frontotemporal dementia. Cogn Neuropsychiatry 2001;6:63-77.

10. Crutch SJ, Lehmann M, Schott JM, et al. Posterior cortical atrophy. Lancet Neurol 2012:11:170-8.

11. Kitzinger J. Qualitative research. Introducing focus groups. BMJ 1995;311:299-302.

12. Owsley C, McGwin G, Scilley K, et al. Perceived barriers to care and attitudes about vision and eye care: focus groups with older African Americans and eye care providers. Invest Ophthalmol Vis Sci 2006;47:2797-802

13. Lacey J, Cate H, Broadway DC. Barriers to adherence with glaucoma medications: a qualitative research study. Eye 2009;23:924-32.

14. Laine C, Davidoff F, Lewis CE, et al. Important elements of outpatient care: a comparison of patients' and physicians' opinions. Ann Intern Med 1996;125:640-5.

15. University College. London rare dementias group - PCA support group. www.raredementiasupport.org

16. UCL. Queen Square tests for visual and cognitive deficits (Green Book and Blue Book) are available here. https://onlinestore.ucl.ac. uk/product-catalogue/faculty-of-brain-sciences-c07/ucl-instituteof-neurology-d07/d07-the-queen-square-screening-test-for-visualdeficits

17. Tong A, Sainsbury P, Craig J. Consolidated criteria for reporting qualitative research (COREQ): a 32-item checklist for interviews and focus groups. Int J Qual Health Care 2007;19:349-57.

18. O'Brien BC, Harris IB, Beckman TJ, et al. Standards for reporting qualitative research: a synthesis of recommendations. Acad Med 2014;89:1245

19. Bowen M, Edgar DF, Hancock B, et al. The Prevalence of Visual Impairment in People with Dementia (the PrOVIDe study): a cross-sectional study of people aged 60-89 years with dementia and qualitative exploration of individual, carer and professional perspectives. Health Services and Delivery Research 2016;4:1-200.

20. Pope C, Ziebland S, Mays N. Qualitative research in health care. Analysing qualitative data. BMJ 2000;320:114-6.

21. Glen FC, Baker H, Crabb DP. A qualitative investigation into patients views on visual field testing for glaucoma monitoring. BMJ Open 2014;4:e003996.

22. Amsler Chart. American macular degeneration foundation - Amsler Chart. https://www.macular.org/amsler-chart

23. Pelak VS, Smyth SF, Boyer PJ, et al. Computerized visual field defects in posterior cortical atrophy. Neurology 2011;77:2119-22.

24. Faes L, Bodmer NS, Bachmann LM, et al. Diagnostic accuracy of the Amsler grid and the preferential hyperacuity perimetry in the screening of patients with age-related macular degeneration: systematic review and meta-analysis. Eye 2014;28:788-96.

25. Beh SC, Muthusamy B, Calabresi $P$, et al. Hiding in plain sight: a closer look at posterior cortical atrophy. Pract Neurol 2015;15. 